\title{
Facile and Efficient Post-synthetic Tritium Labelling Method Catalyzed by Pd/C in HTO
}

\author{
Tomohiro Maegawa, ${ }^{\dagger}$ Kosaku Hirota $^{\dagger}{ }^{\text {Kenjiro Tatematsu, }}{ }^{\ddagger}$ Yukio Mori, ${ }^{\ddagger}$ Hironao Sajiki ${ }^{*}{ }^{\dagger}$ \\ ${ }^{\dagger}$ Laboratory of Medicinal Chemistry, Gifu Pharmaceutical University \\ ${ }^{\ddagger}$ Laboratory of Radiochemistry, Gifu Pharmaceutical University \\ 5-6-1, Mitahora-higashi, Gifu, 502-8585, Japan \\ sajiki@gifu-pu.ac.jp
}

Tel: +81-58-237-3931, Fax: +81-58-237-5979

\section{Supporting Information}

\section{Contents}

Page S2: General.

Page S2-4: Experimental Procedures and Spectral Data.

Page S5: $\quad$ The deuteration data of the substrates in Table 2.

Page S6: $\quad$ References.

Page S7-13: $\quad{ }^{1} \mathrm{H}$ NMR, ${ }^{2} \mathrm{H}$ (D) NMR, ${ }^{13} \mathrm{C}$ NMR and Mass spectroscopy charts for ibuprofen- $d$ and acetaminophen- $d$. 


\section{General:}

$10 \% \mathrm{Pd} / \mathrm{C}$ was purchased from Aldrich. All of the substrates were purchased from commercial sources and used as received. HTO was prepared by diluting the tritiated water (37 MBq/g; purchased from MORAVEK) with distilled water.

${ }^{1} \mathrm{H},{ }^{2} \mathrm{H}$ and ${ }^{13} \mathrm{C}$ NMR spectra were measured with trimethylsilane or residual protiated solvent used as a references. Purity were determined by HPLC analysis. Radioactivity measurement were made on a liquid scintillation analyzer.

\section{Experimental Procedure:}

Caution: The compounds containing tritium atoms are hazardous, especially in the case of inhalation. All experiments were conducted with groves in the fume food in the control area for radiation. And the reaction should be taken care of the internal pressure which rise up to ca. $2.5 \mathrm{~atm}$ in the sealed tube at $160^{\circ} \mathrm{C}$.

\section{Typical Procedure for the Tritiation Reactions Using Pd/C-HTO-H at Room Temperature.}

\section{(Scheme 1)}

A suspension of diphenylmethane $(1.0 \mathrm{mmol})$ and $10 \% \mathrm{Pd} / \mathrm{C}$ (10 wt\% of substrate) in HTO (0.5 mL) were stirred at room temperature in a test tube under $\mathrm{H}_{2}$ atmosphere for $72 \mathrm{~h}$. The mixture was diluted with ether $\left(20 \mathrm{~mL}\right.$ ) and filtered by a membrane filter (Millipore, Millex $\left.{ }^{\mathrm{R}}-\mathrm{LH}, 0.45 \mu \mathrm{m}\right)$. The filtrate was extracted with ether (20 mL x 2). The combined etheral layer was washed with $\mathrm{H}_{2} \mathrm{O}$ and sat. $\mathrm{NaCl}$ aq and dried with $\mathrm{MgSO}_{4}$, then concentrated in vacuo. Measurements of the specific activity of the substrate were made on a liquid scintillation analyzer.

[ ${ }^{3}$ H]Diphenylmethane (1). Yield: $100 \%$ (99.0\% pure by HPLC), specific activity: $1.28 \mathrm{kBq} / \mathrm{mmol}$ (using $2.22 \mathrm{kBq} / \mathrm{mmol}$ of HTO) 


\section{Typical Procedure for the Tritiation Reactions Using Pd/C-HTO-H $\mathrm{H}_{2}$ at $160{ }^{\circ} \mathrm{C}$. (Table 1 and}

\section{Table 2, Entry 1)}

A suspension of substrate (2-4) (0.5 mmol) and 10\% Pd/C (10 wt\% of substrate) in HTO (2 mL) were stirred at $160{ }^{\circ} \mathrm{C}$ in a sealed tube under $\mathrm{H}_{2}$ atmosphere for $24 \mathrm{~h}$. The mixture was diluted with ether (20 $\mathrm{mL}$ ) and filtered by a membrane filter (Millipore, Millex ${ }^{\mathrm{R}}-\mathrm{LH}, 0.45 \mu \mathrm{m}$ ). The filtrate was extracted with ether (20 mL x 2). The combined etheral layer was washed with $\mathrm{H}_{2} \mathrm{O}$ and sat. $\mathrm{NaCl}$ aq and dried with $\mathrm{MgSO}_{4}$, then concentrated in vacuo. Measurements of the specific activity of substrate were made on a liquid scintillation analyzer.

[ ${ }^{3}$ H]3-Phenylpropionic acid (2). Yield: $98 \%$ (99.3 \% pure by HPLC), specific activity: 1.82 $\mathrm{kBq} / \mathrm{mmol}$ (using $0.67 \mathrm{kBq} / \mathrm{mmol}$ of HTO)

[ ${ }^{3}$ H]4-Propylbenzoic acid (3). Yield: 87 \% (99.9 \% pure by HPLC), specific activity: 5.50 kBq/mmol (using $2.22 \mathrm{kBq} / \mathrm{mmol}$ of HTO)

[ ${ }^{3}$ H]Ibuprofen (4). Yield: 96 \% (99.0 \% pure by HPLC), specific activity: 3.42 kBq/mmol (using $0.67 \mathrm{kBq} / \mathrm{mmol}$ of HTO)

\section{Typical Procedure for the Tritiation Reactions Using Pt/C-HTO-H2 at $160{ }^{\circ} \mathrm{C}$. (Table 2, Entry}

\section{2)}

A suspension of acetaminophen $(0.5 \mathrm{mmol})$ and $5 \% \mathrm{Pt} / \mathrm{C}(20 \mathrm{wt} \%$ of substrate) in HTO (2 mL) were stirred at $160{ }^{\circ} \mathrm{C}$ in a sealed tube under $\mathrm{H}_{2}$ atmosphere for $24 \mathrm{~h}$. The mixture was diluted with ether (20 $\mathrm{mL}$ ) and filtered by a membrane filter (Millipore, Millex ${ }^{\mathrm{R}}-\mathrm{LH}, 0.45 \mu \mathrm{m}$ ). The filtrate was extracted with ether (20 mL x 2). The combined etheral layer was washed with $\mathrm{H}_{2} \mathrm{O}$ and sat. $\mathrm{NaCl}$ aq and dried with $\mathrm{MgSO}_{4}$, then concentrated in vacuo. Measurements of the specific activity of substrate were made on a liquid scintillation analyzer. 
[33]Acetaminophen (5). Yield: $72 \%$ (99.9 \% pure by HPLC), specific activity: $4.26 \mathrm{kBq} / \mathrm{mmol}$ (using $2.22 \mathrm{kBq} / \mathrm{mmol}$ of $\mathrm{HTO}$ )

Typical Procedure for the Tritiation Reactions Using Pd/C-HTO- $\mathrm{H}_{2}$ at $110{ }^{\circ} \mathrm{C}$. (Table 2, Entries 3 and 4$)$

A suspension of substrate (6 and 7) $(0.5 \mathrm{mmol})$ and $10 \% \mathrm{Pd} / \mathrm{C}(10 \mathrm{wt} \%$ of substrate) in HTO (2 mL) were stirred at $110{ }^{\circ} \mathrm{C}$ with sealed reflux condenser under ordinary $\mathrm{H}_{2}$ pressure ${ }^{1}$ for $24 \mathrm{~h}$. The mixture was diluted with distilled water $(10 \mathrm{~mL})$ and filtered by a membrane filter (Millipore, Millex ${ }^{\mathrm{R}}$-LH, 0.45 $\mu \mathrm{m})$. The filtrate was concentrated in vacuo. Measurements of the specific activity of substrate were made on a liquid scintillation analyzer.

[ ${ }^{3}$ H]Adenosine (6). Yield: 99 \%, specific activity: $0.70 \mathrm{kBq} / \mathrm{mmol}$ (using $2.22 \mathrm{kBq} / \mathrm{mmol}$ of HTO)

[ ${ }^{3} \mathbf{H}$ ]Phenylalanine (7). Yield: $94 \%$, specific activity: $0.62 \mathrm{kBq} / \mathrm{mmol}$ (using $2.22 \mathrm{kBq} / \mathrm{mmol}$ of HTO) 
Table S1. The deuteration data of the substrates in Table 2

$$
\text { Substrate } \underset{\mathrm{H}_{2}, 160^{\circ} \mathrm{C} \text { or } 110^{\circ} \mathrm{C}, 24 \mathrm{~h}}{\stackrel{10 \% \mathrm{Pd} / \mathrm{C} \text { or } 5 \% \mathrm{Pt} / \mathrm{C}, \mathrm{D}_{2} \mathrm{O}}{\longrightarrow}} \text { Product- } \boldsymbol{d}_{\boldsymbol{n}}
$$

Entry Substrate

Ibuprofen-d (8) (Table S1, entry 1): ${ }^{1} \mathrm{H}$ NMR (400 MHz, $\mathrm{CD}_{3} \mathrm{OD}$ ): $\delta=0.74$ (brs, $0.32 \mathrm{H}$ ), 1.30 (brs, $0.14 \mathrm{H}), 1.69$ (brs, $0.04 \mathrm{H}), 2.32$ (brs, $0.09 \mathrm{H}), 3.56$ (m, $0.06 \mathrm{H}), 6.99$ (d, $J=8.2 \mathrm{~Hz}, 1.44 \mathrm{H}), 7.11$ (d, $J$ $=8.2 \mathrm{~Hz}, 1.44 \mathrm{H}) .{ }^{2} \mathrm{H}$ NMR $\left(60.7 \mathrm{MHz}, \mathrm{CH}_{3} \mathrm{OH}\right): \delta=0.82,1.37,1.75,2.39,3.61,7.11,7.23 .{ }^{13} \mathrm{C} \mathrm{NMR}$ (100.4 MHz, $\left.\mathrm{CD}_{3} \mathrm{OD}\right): \delta=128.1,128.2,130.1,130.2,139.7,141.5,178.5$. (The peaks of alkyl carbons could not be detected. See the following NMR charts.) MS (EI): $m / z=2.2 \% \mathrm{~d}_{10}, 9.5 \% \mathrm{~d}_{11}, 26.2 \% \mathrm{~d}_{12}$, $37.3 \% \mathrm{~d}_{13}, 17.0 \% \mathrm{~d}_{14}, 4.8 \% \mathrm{~d}_{15}, 1.8 \% \mathrm{~d}_{16}, 1.2 \% \mathrm{~d}_{17}$.

Acetaminophen-d (9) (Table S1, entry 2): ${ }^{1} \mathrm{H}$ NMR (400 MHz, DMSO-d 6 ): $\delta=2.00$ (s, $\left.2.79 \mathrm{H}\right), 6.69$ (Brs, $0.05 \mathrm{H}), 7.35$ (brs, $0.05 \mathrm{H}), 9.14$ (s, $0.99 \mathrm{H}), 9.67$ (s, $0.99 \mathrm{H}) .{ }^{2} \mathrm{H}$ NMR $\left(60.7 \mathrm{MHz}, \mathrm{CH}_{3} \mathrm{OH}\right): \delta=$ 2.03, 2.06, 6.74, 7.31. ${ }^{13} \mathrm{C}$ NMR (100.4 MHz, $\left.\mathrm{CD}_{3} \mathrm{OD}\right): \delta=23.5,116.3(\mathrm{~m}), 123.5$ (m), 131.5, 155.3, 171.3. MS (EI): $\mathrm{m} / \mathrm{z}=1.1 \% \mathrm{~d}_{2}, 10.6 \% \mathrm{~d}_{3}, 62.0 \% \mathrm{~d}_{4}, 19.8 \% \mathrm{~d}_{5}, 4.6 \% \mathrm{~d}_{5}, 1.8 \% \mathrm{~d}_{6}$. 


\section{References:}

1) An excess amount of $\mathrm{H}_{2}$ gas depress the deuterium efficiency at the benzylic position, see: (a) Sajiki, H.; Hattori, K.; Aoki, F.; Yasunaga, K.; Hirota, K. Synlett 2002, 1149-1151; (b) Maegawa, T.; Akashi, A.; Esaki, H.; Aoki, F.; Sajiki, H.; Hirota, K. Synlett 2005, 845-847.

2) The deuteration of ibuprofen sodium salt has been reported, see: Sajiki, H.; Aoki, F.; Esaki, H.; Maegawa, T.; Hirota, K. Org. Lett. 2004, 6, 1485-1487.

3) Sajiki, H.; Ito, N.; Esaki, H.; Maesawa, T.; Maegawa, T.; Hirota, K. Tetrahedron Lett. 2005, 46, 6995-6998.

4) Sajiki, H.; Esaki, H.; Aoki, F.; Maegawa, T.; Hirota, K.; Synlett 2005, 1385-1388. 


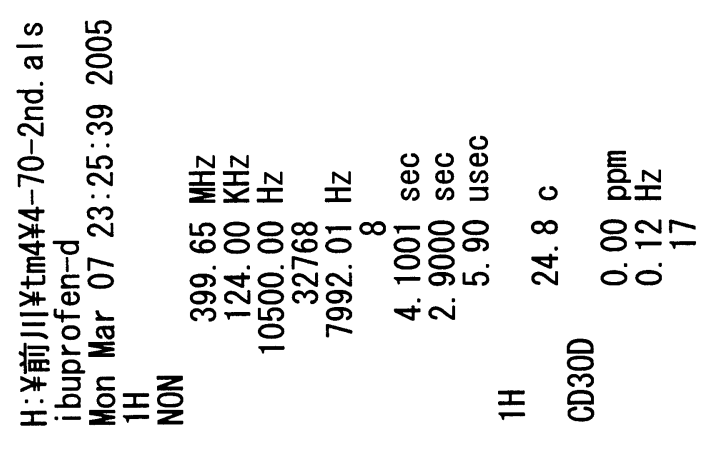

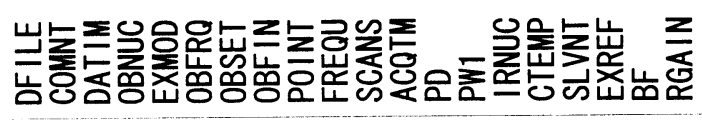

$Z t L^{\circ} 0$

$862 \%$

$989 \%$

SIE 7

$\nabla 2 \tau$

s18.

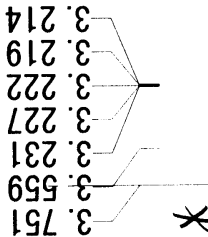

$96 z$

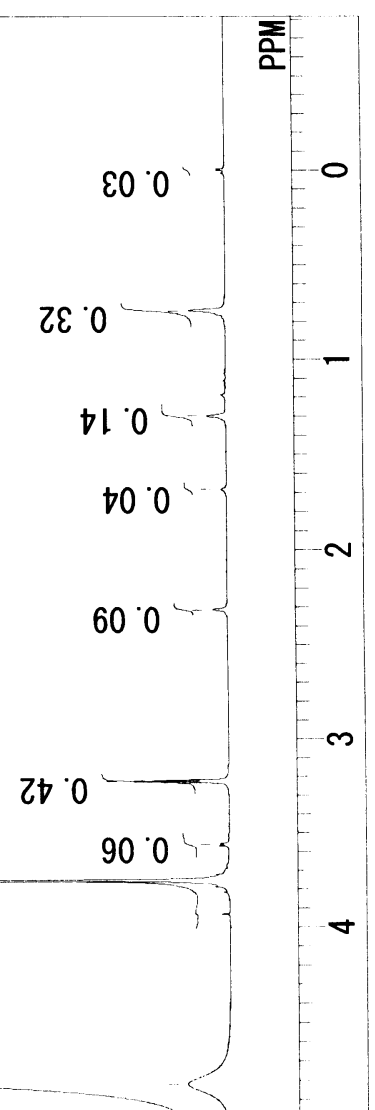

$\frac{0}{5}$

เ

$\frac{u}{4}$

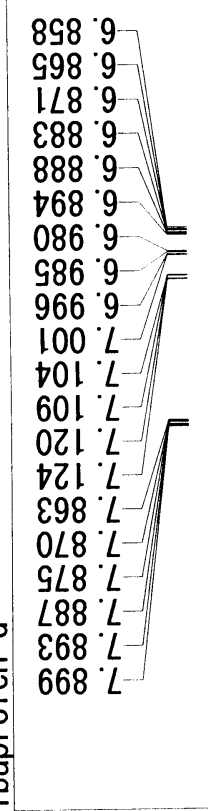

$0 Z 9$

*

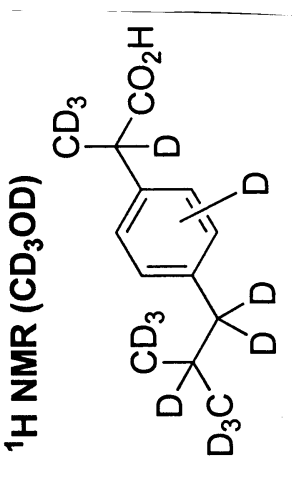

100

$00 \%$

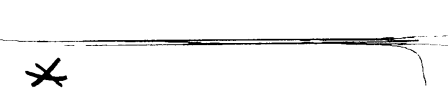

$\infty$

2 


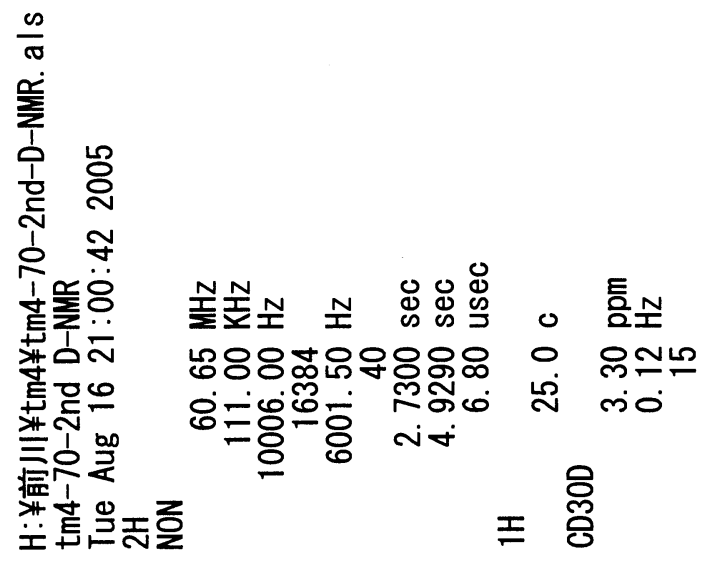

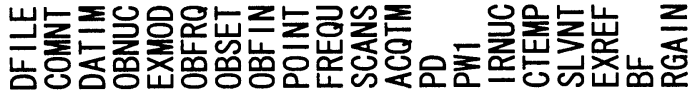

$00 \% 9$

$\varepsilon Z 8^{\circ} 0$

$0 \varepsilon 1 \cdot$

$I L \varepsilon^{\prime} !$

$S t L '$

69 乙

$00 \varepsilon^{\circ} \varepsilon$

$\mathcal{S L t} \varepsilon$

$\varepsilon 19^{\circ} \varepsilon$

$Z G L$

$L L ' Z$

$06 \varepsilon z$

$86 \cdot 1$

$919 \cdot$

$26^{\circ} 0$

$88^{\circ} 0$

$126^{\circ}$

†6 0

$601{ }^{\circ} L$

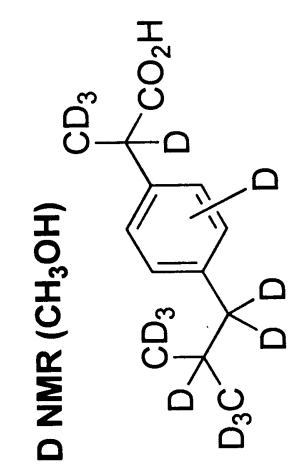

$\varepsilon t^{\circ} 0$

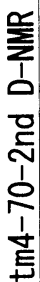

$0 \varepsilon Z^{\circ} L$

立 


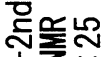

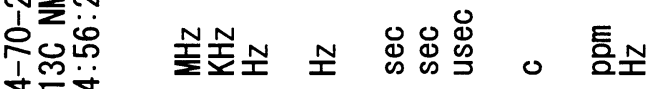

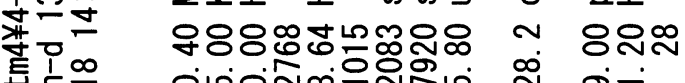

*

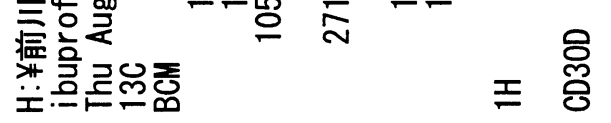

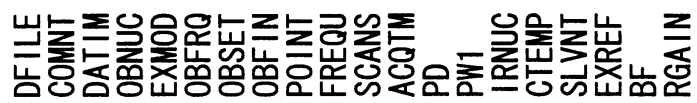

$99 \varepsilon^{\circ} 8 t$

$089.8 \mathrm{t}$

$76 L$ 8t

000.67

$\rightarrow 1260$

8276

27967

$998^{\circ} 6 \mathrm{t}$

801821

$\varepsilon Z Z$ '8Z1

GZ1 $0 \varepsilon$

$0 \supset 0^{\circ} 0 \varepsilon$

$\angle 996 \varepsilon$ ।

LZS $|+|$

$\operatorname{Ztg} 8 L 1$
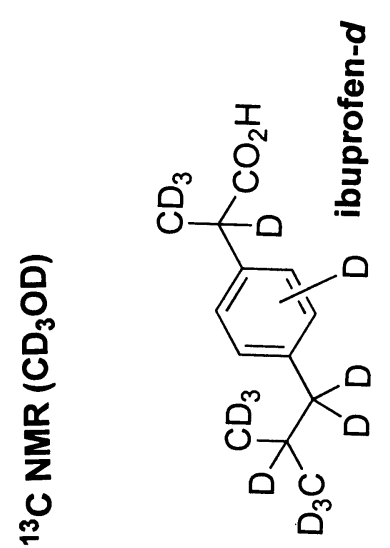


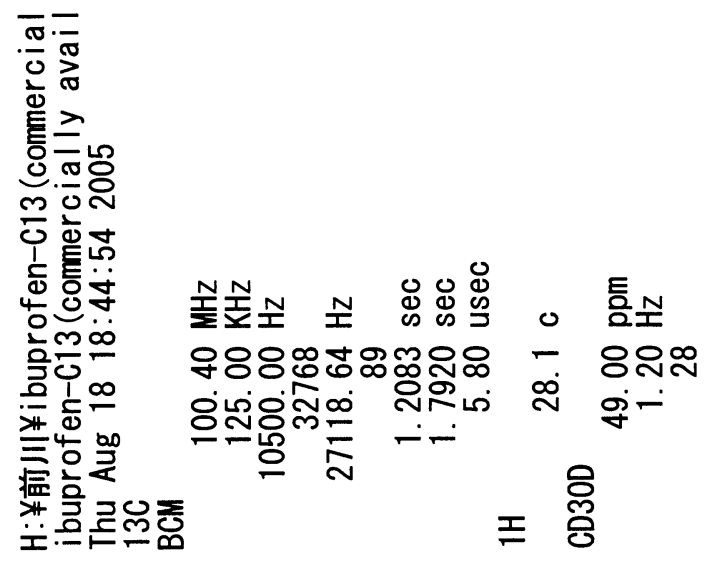

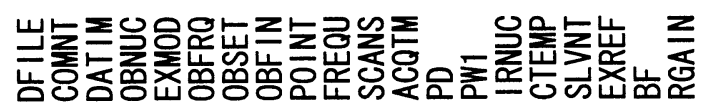

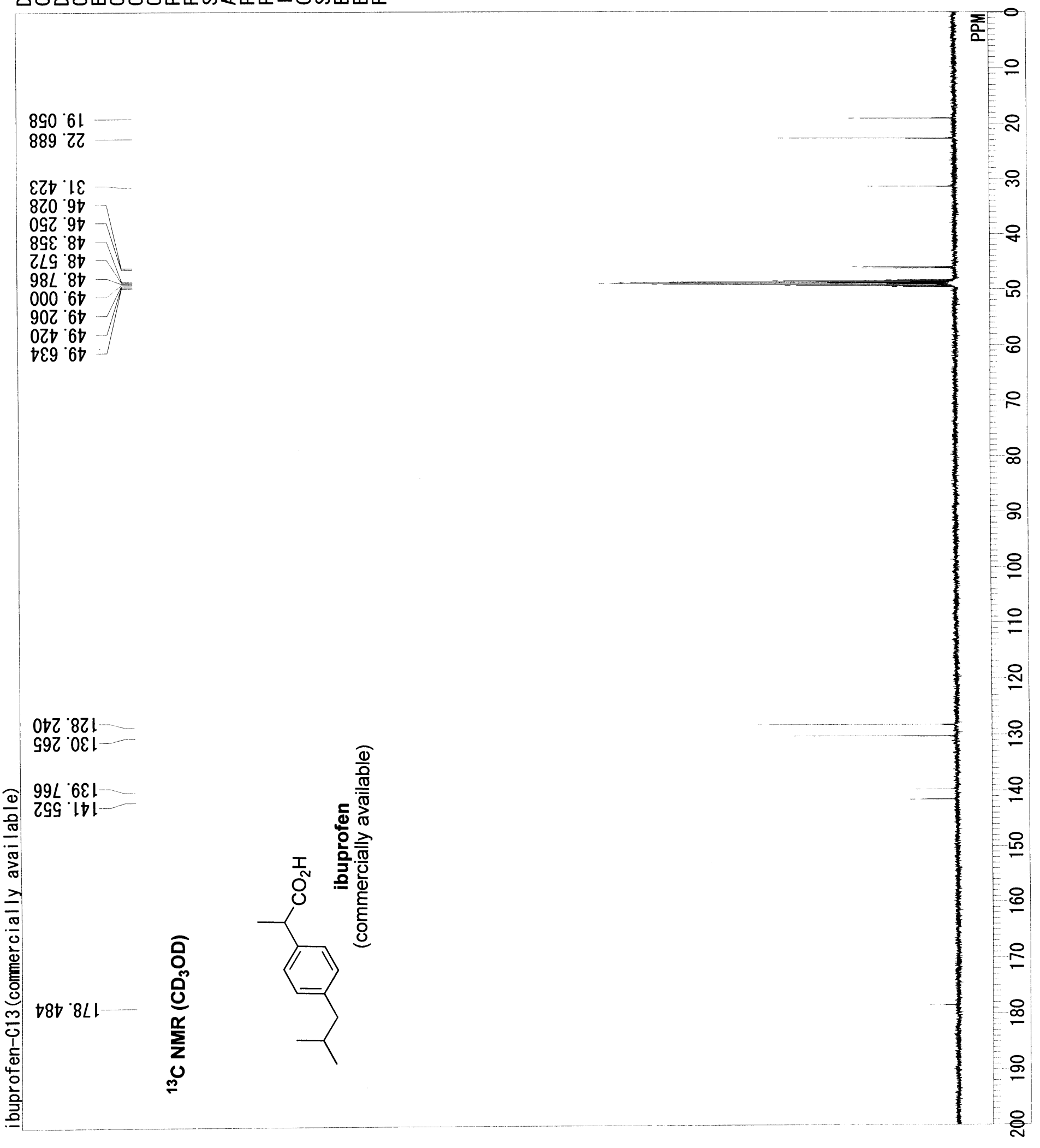


$\frac{n}{\ddot{n}}$<smiles>[O]C([O])(O)C(O)(O)c1ccc(C(O)(O)C([O])([O])O[Na])cc1O</smiles>

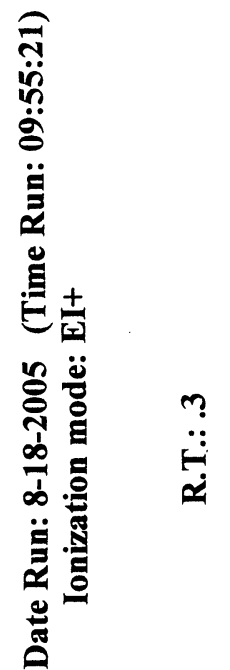

$\frac{a}{2}$

ᄋิ-

今.

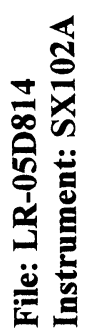

붑요

$\stackrel{ \pm}{\Xi}$

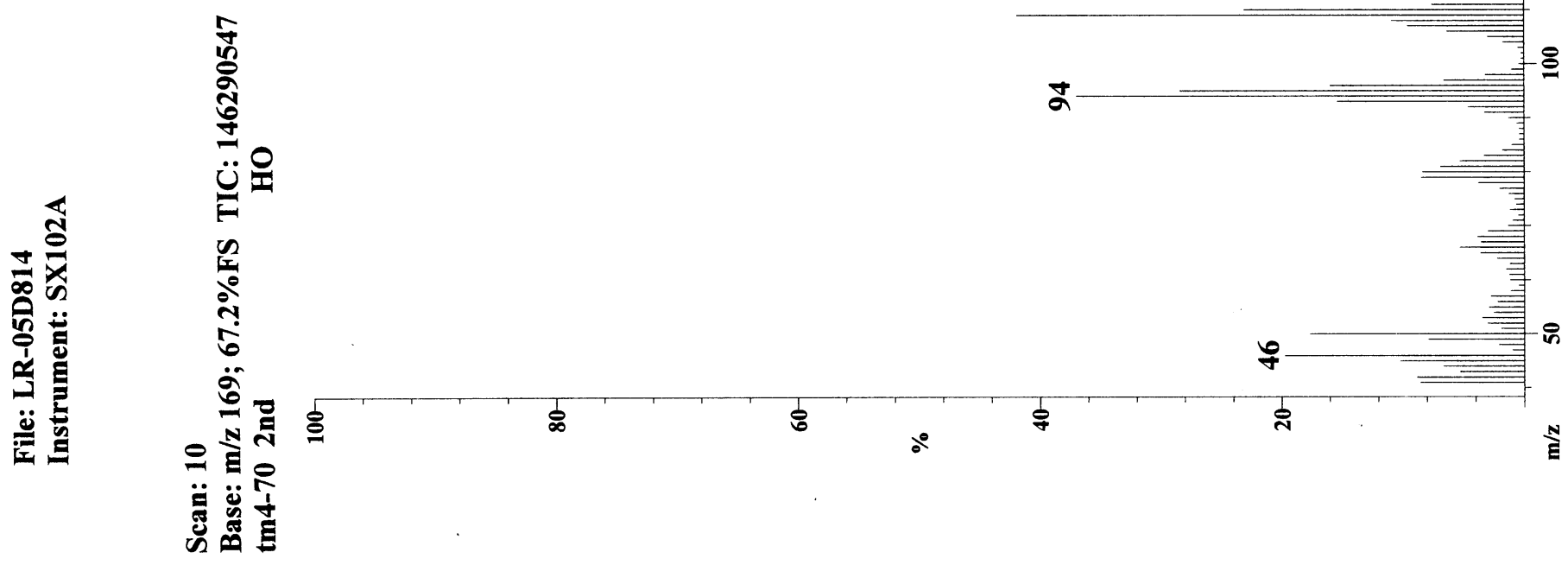




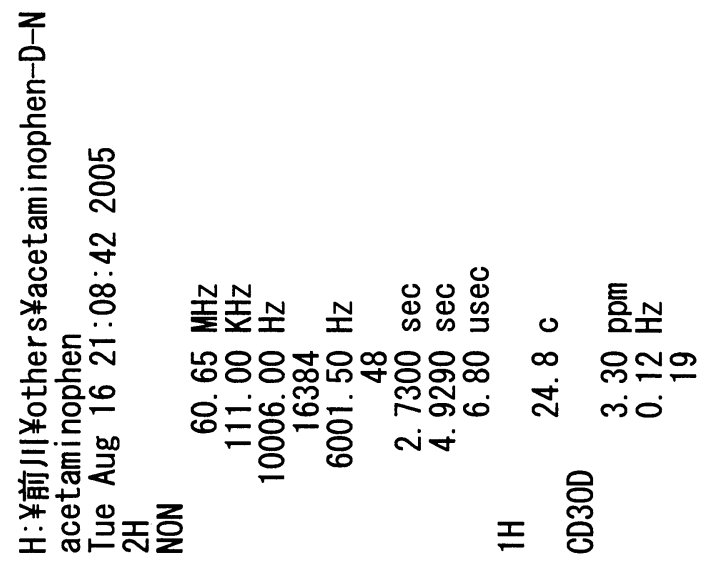

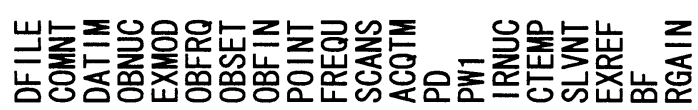

$820 \%$

$890 \%$

$00 \varepsilon^{\circ} \varepsilon$

$G \angle t^{\circ} \mathcal{E}$

$6 L 8^{\circ} \circ$

$96^{\circ} \varepsilon$

乙๐L 9

$00 \%$

$10 \%$

$80 \varepsilon^{\circ} L$

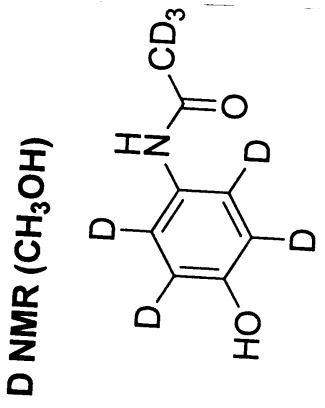




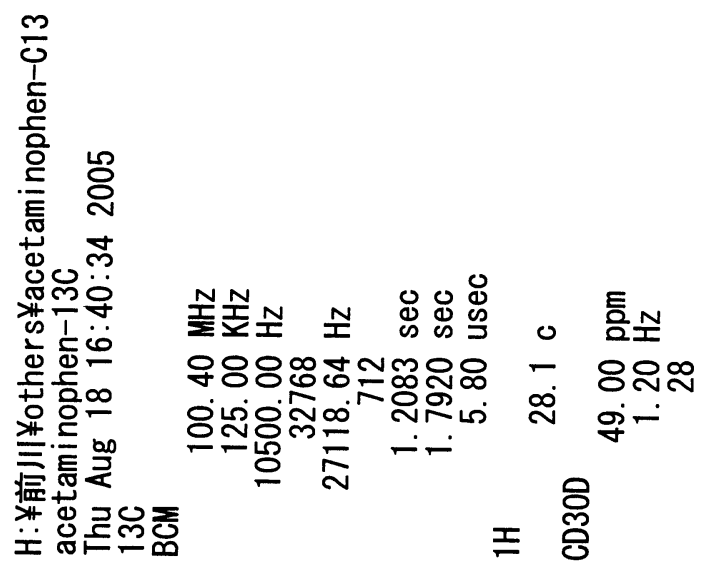

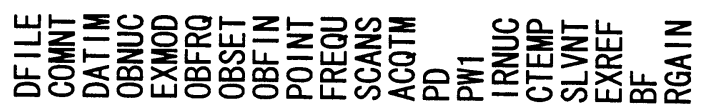

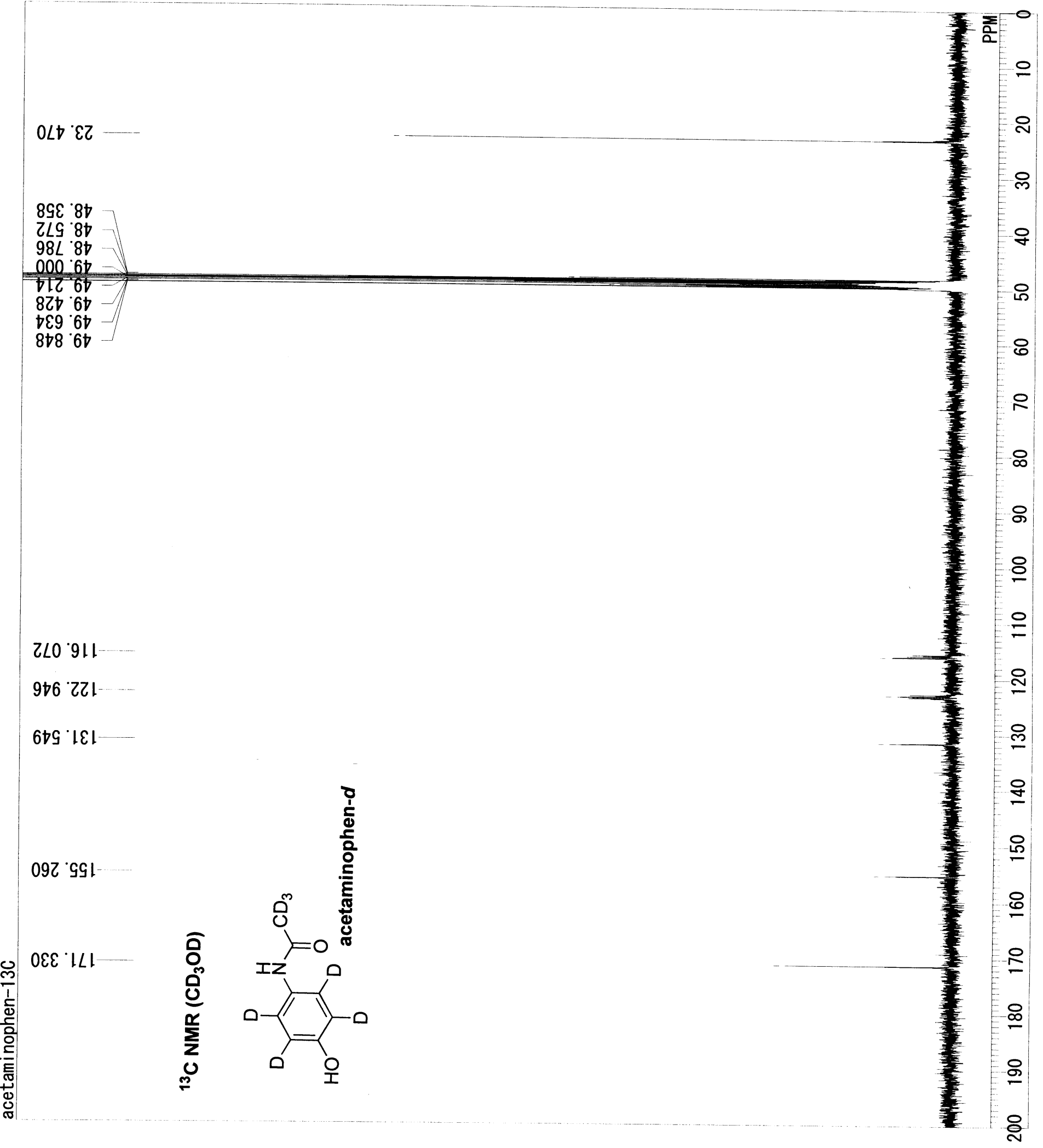


产㐫:

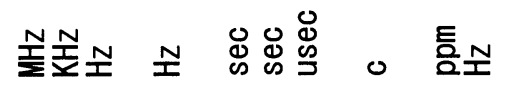

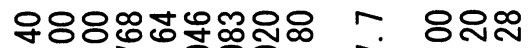

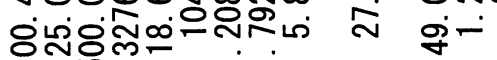

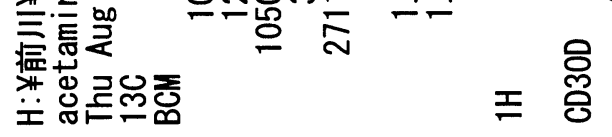

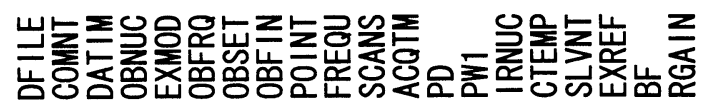

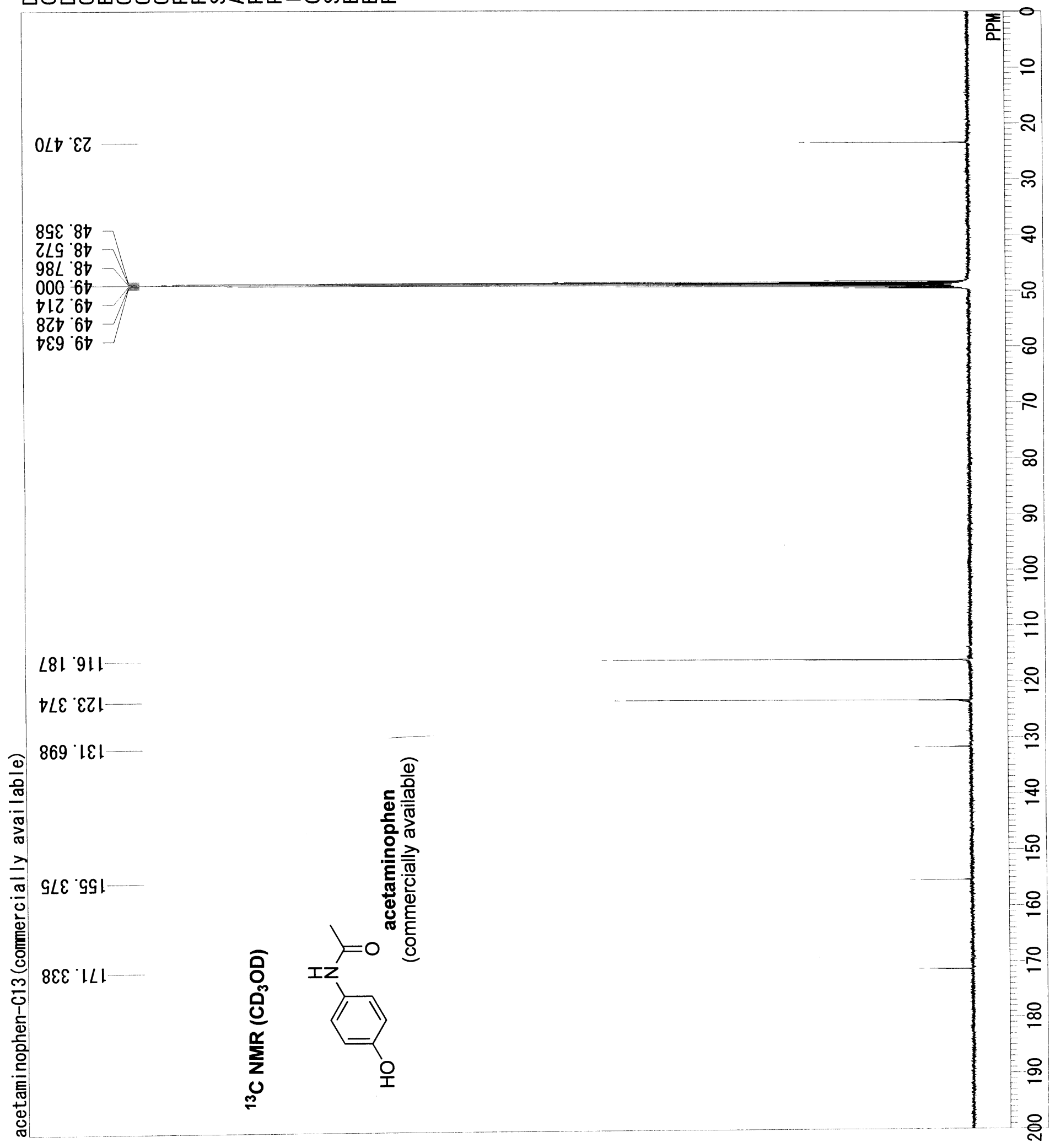




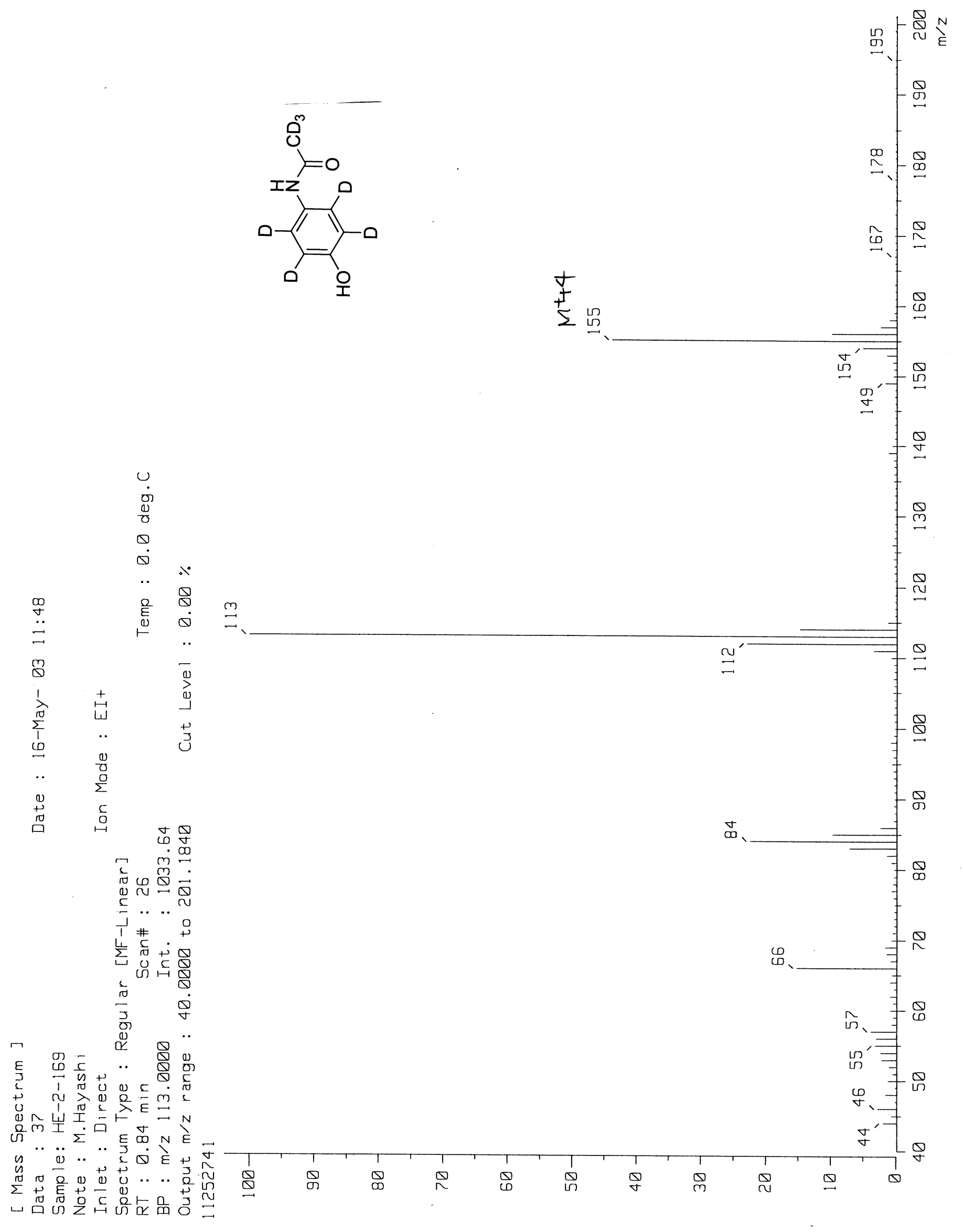

